\title{
Improved Particle Swarm Optimization Algorithm for Optimization of Power Communication Network
}

\author{
Yuhuai Wang, Qihui Wang *, Huixi Zhang, \\ Kang An, Xia Ye and Yaping Sun \\ *Address correspondence to Qihui Wang; \\ wangqihui_xinyi@hotmail.com \\ Qianjiang College, Hangzhou Normal University, \\ Hangzhou 310036, China
}

\begin{abstract}
Based on particle swarm optimization (PSO) algorithm and its power system reactive power optimization method to in-depth study and research proposed a new hybrid particle swarm optimization algorithm (HPSO). Algorithm combines the differential evolution algorithm and simulated annealing algorithm and particle swarm optimization algorithm, in particle searching optimal except for tracking individual and global, and tracks produced by particle information difference of the three value. At the same time, when the particle search space of one dimension speed lower than the setting value will be re initialized the dimensional particle velocity and the particle of differential evolution mutation. For the crossover and mutation operations, new solution may be worse than the original solution to, the introduction of simulated annealing algorithm, the metropolis rule in a certain extent accept bad solutions, allows the target function in a certain degree of deterioration, practical calculation is not according to the probability to choose the poor solution, but rather the judgment target function difference is less than allows the target function deterioration range. Hybrid particle swarm optimization algorithm combines the advantages of particle swarm optimization algorithm, differential evolution algorithm and simulated annealing algorithm, to maintain the diversity of particles, has very strong practicability.
\end{abstract}

Keywords: Particle swarm optimization; power communication network; simulated annealing; differential evolution total quantity of knowledge

\section{Introduction}

Particle swarm optimization algorithm based on swarm intelligence algorithm is a stochastic optimization theory, is a new heuristic algorithm. The algorithm parameter setting is simple, strong robustness, easy to implement, with global searching optimum and converge fast, in order to improve the performance of the algorithm, an improved PSO algorithm is endless, to apply in different engineering fields. In recent years, with the development of communication technology, in order to meet the power system safe, stable, efficient production requirements, in order to meet the electric power business operations to the market demand, the development of electric power communication network is very fast. Many new communication equipment, communication system, such as digital SPC, SDH fiber loop, SDH microwave, ATM and WDM, have the influx of electric power communication network, the network features change rapidly [3]. As a special communication network industry, electric power communication network is needed with the development of power system and the gradual formation and development. It is mainly used to alleviate public development caused by slow [4]. Some special communication demand of electric power department communication ability is insufficient and fill the public network is difficult to meet the specialized production, to 
ensure the normal power efficiently, and promote the development of the national economy $[5,6]$.

Improve voltage quality first, to ensure that power is equal to the power grid operation equipment production and consumption of reactive power balance, namely the system reactive power reactive power output and power grid load and equipment in the operating state of rated voltage demand. Reactive power is insufficient, generally low voltage system, voltage lower limit operation, is easy to be affected by voltage disturbance influence the occurrence of major accidents, the impact of the economy and the stability of the system operation; reactive power surplus, system voltage will too high, is not conducive to the safe and stable operation of the equipment, and increase the system active power loss, decline in the quality of power supply voltage, operating costs is also a corresponding increase. System reactive power of unreasonable flow will lead to system voltage mutation, the net loss is increased, the transmission and increased maintenance costs. Therefore, reasonable distribution of reactive power in the system is of great significance to the security and stability of power system, the quality of economic operation. Therefore, to the rational allocation of reactive power compensation device, reactive power balanced to ensure power system.

With the gradual increase of the grid scale, residents demand more and more high, power system operation stability and economy are increasingly affected by the people's attention. Without reactive power optimization as power system security, stability and economic operation of the most important means of control, to ensure the safe and reliable operation of the power system under the premise, how scientific allocation of reactive power resources, effectively reduce the loss of the system, further improve the system performance, being subject to domestic and foreign scholars have been solved for many years. Firstly, to the domestic and foreign power system without reactive power optimization research present situation to carry on the research and analysis the no reactive power control method, and established the system of minimum network loss as the objective function of the mathematical model. The basic particle swarm algorithm were research and analysis, which combines the differential evolution algorithm and simulated annealing algorithm and particle swarm optimization algorithm, a new hybrid particle swarm optimization algorithm (HPSO) is proposed. The HPSO algorithm combines the advantages of three algorithms, has good search performance. For control variables both continuous variables and discrete variables characteristics, adopt hybrid coding method to encode the variables. At the same time, the mapping and four to five warping method dealing with the coexistence of continuous and discrete variables homes.

\section{The Relevant Thoery}

\section{A. Mathematical description of PSO algorithm}

The number of particles in the population is population size $\mathrm{N}$, search space for $\mathrm{D}$ dimension, and each particle contains a $\mathrm{D}$ dimension position vector and a $\mathrm{D}$ dimension velocity vector. $X_{i}=\left(X_{i 1}, X_{i 2}, \ldots, X_{i D}\right)^{T}$ to represent the position vector of the i particles in a population, $V_{i}=\left(V_{i 1}, V_{i 2}, \ldots, V_{i D}\right)^{T}(i=1,2, \ldots, N)$ to represent the velocity vector of the i particles, and $P_{i}=\left(P_{i 1}, P_{i 2}, \ldots, P_{i D}\right)^{T}$ to represent the best position for particle searching. The whole population is represented by $\mathrm{g}, P_{g}=\left(P_{g 1}, P_{g 2}, \ldots, P_{g D}\right)^{T}$ to represent the best position for all particles searching in a population, which is adjusted the position of particles in the population and searching speed. The particles are iterated by the following formula:

$V_{i j}^{k+1}=V_{i j}^{k}+c_{1} r_{1 j}\left(P_{i j}^{k}-x_{i j}^{k}\right)+c_{2} r_{2 j}\left(P_{i j}^{k}-x_{i j}^{k}\right)$ 
$X_{i j}^{k+1}=X_{i j}^{k}+V_{i j}^{k+1}$

In which $i=1,2, \ldots, N, \mathrm{j}$ to represent the dimension of particles in search space, $\mathrm{c} 1, \mathrm{c} 2$ are acceleration factors, generally value between 0-2. c1 adjust the step size of the particle local search, c2 adjust the step size of the particle global search, $r_{1} \sim u(0,1), r_{2} \sim u(0,1)$ are randomly initialized functions. The particle velocity $V_{i j}$ is usually limited $V_{i j} \in\left[-V_{\max }, V_{\max }\right]$ to reduce the probability of particles leaving the search space, it is usually associated with position constraints. Such as position information constraint is $\left[-X_{\max }, X_{\max }\right]$, then set up $V_{\max }=k X_{\max }, 0.1 \leq k \leq 1.0$

\section{B. PSO algorithm flow}

Autonomy refers to an entity with its own activity in an environment, its simple, usually does not need to have a senior intelligence, however, is an advanced smart to reach their activities the activities of the clusters shown, we call this kind of activities for the swarm intelligence. Swarm intelligence is a kind of biological system and the social system. The simulation system makes use of local information to generate the group behavior cannot be predicted.

$$
\begin{aligned}
& v_{i}^{j}(t+1)=v_{i}^{j}(t)+\operatorname{rand}_{1}^{j}\left(\text { pbest }_{i}^{j}-x_{i}^{j}(t)\right)+\operatorname{rand}_{2}^{j}\left(\text { gbest }_{i}^{j}-x_{i}^{j}(t)\right) \\
& x_{i}^{j}(t+1)= \begin{cases}1, & \text { if } \operatorname{rand}()<\operatorname{sig}\left(v_{i}^{j}(t+1)\right) \\
0, & \text { if } \operatorname{rand}() \geq \operatorname{sig}\left(v_{i}^{j}(t+1)\right)\end{cases}
\end{aligned}
$$

At present most of the improved PSO algorithm with adaptive strategy is improved based on adaptive linear, proposed by Shi and Eberhart decreasing inertia weight strategy can be stated as follows:

$$
\omega=\omega_{\max }-\left(\omega_{\max }-\omega_{\min }\right) \frac{g}{G}
$$

The value of inertia weight according to the type of change:

$$
\omega(t)=\left\{\frac{(T-t)^{n}}{t^{n}}\right\}\left(\omega_{\max }-\omega_{\min }\right)+\omega_{\text {min }}
$$

Particle swarm optimization (PSO) algorithm because of its rule is simple, easy to operate, adjustable parameter is easy to control, fast convergence speed, etc., but it in search of the optimization process is easy to fall into local optimum, resulting in "premature" phenomenon. The PSO algorithm flow chart was shown in Figure 1. 


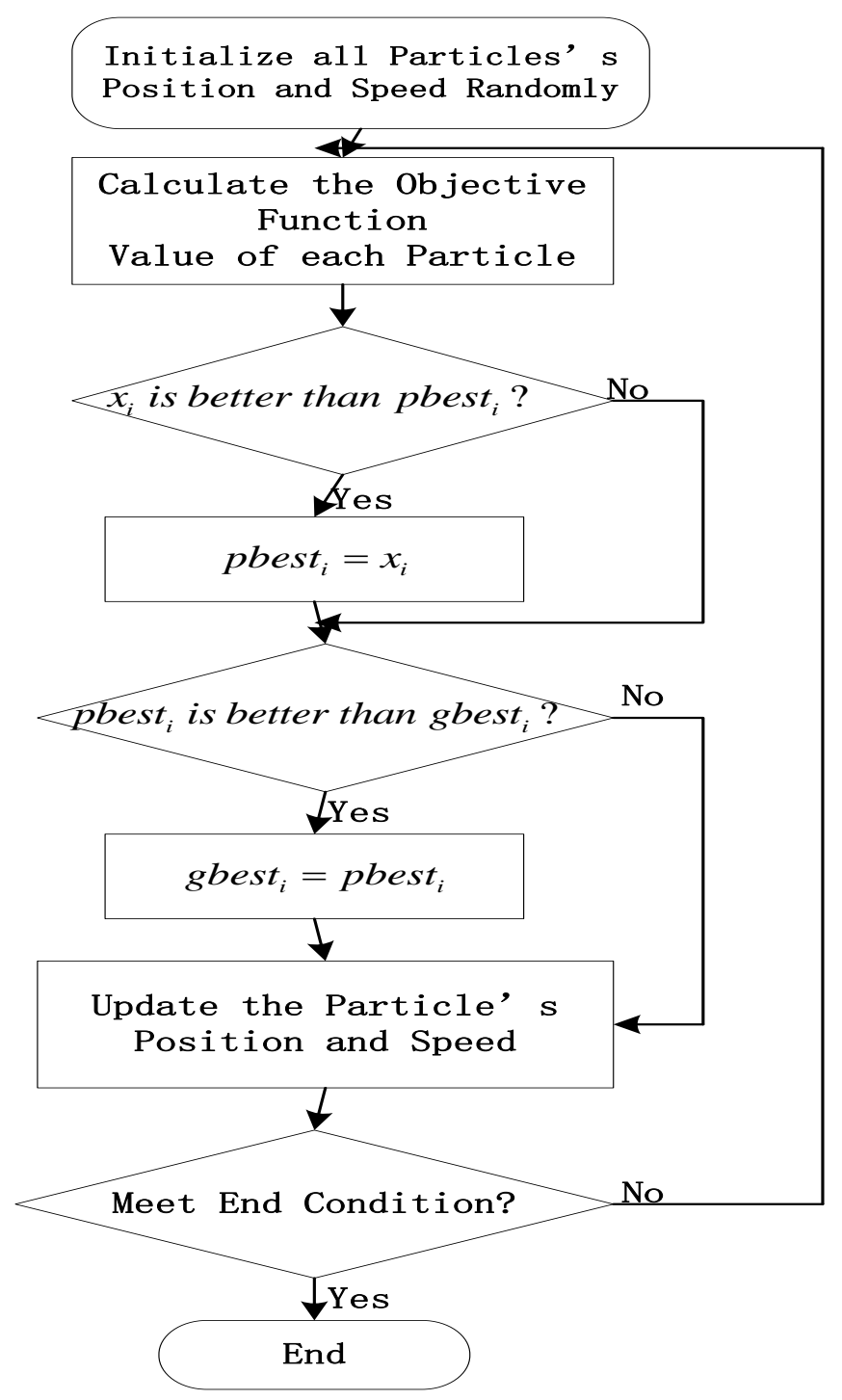

Figure 1. The PSO Algorithm Flow Chart

\section{Mathematical model of optimization}

Reactive power optimization of power system is according to the system load situation, by adjusting the generator terminal voltage, changing the transformer points joint position and switching of reactive power compensation devices, and ultimately to reduce the power loss of the system, reduce the voltage limit of and to improve the voltage quality, make the security and stability of power grid and economic power to users.

Adopting the mathematical model of active power loss minimization as the objective function, to Vgi of the output voltage of the generator, transformer secondary side ratio of Tki and capacitance compensation capacity Qgi as control variables, generator reactive power output Qgi and node voltage Vi, as state variables, in the objective function into the penalty function to solve the generator reactive power output Qgi and load node voltage $\mathrm{Vi}$ variable is limit. The objective function is as follows:

$F=\sum_{i=1, j \in k}^{N} G_{k}(i, j)\left[V_{i}^{2}+V_{j}^{2}-2 V_{i} V_{j} \cos \theta_{i j}\right]+\lambda_{1} \sum_{i=1}^{N}\left(\frac{V_{i}-V_{i \min }}{V_{i \max }-V_{\min }}\right)^{2}+\lambda_{2} \sum_{i=1}^{N_{p v}}\left(\frac{Q_{i}-Q_{i \min }}{Q_{i \max }-Q_{\min }}\right)^{2}$ 
In which $N, \quad N_{p v}$ are generator node number and $P V$ node number, $G_{k}(i, j)$ is conductance of branch $k, V_{i}, V_{j}$ are operating voltage of node number $i, j, \theta_{i j}$ is Voltage angle difference between ode number $i$ and $j, \lambda_{1}, \lambda_{2}$ are penalty factor for the more limited, $V_{i \max }, V_{i \text { min }}$ are respectively the upper and lower limits of node voltage $V_{i}, Q_{i \max }, Q_{i \text { min }}$ are respectively the upper and lower limit of motor reactive power $Q_{G i}$, the definitions are as follows:

$$
\begin{gathered}
V_{i \lim }=\left\{\begin{array}{lr}
V_{i \min }, & V_{i}<V_{i \min } \\
V_{i \max }, & V_{i}>V_{i \max } \\
V_{i}, & \text { other }
\end{array}\right. \\
Q_{i \lim }=\left\{\begin{array}{lr}
Q_{i \min }, & Q_{i}<Q_{i \min } \\
Q_{i \max }, & Q_{i}>Q_{i \max } \\
Q_{i}, & \text { other }
\end{array}\right.
\end{gathered}
$$

\section{Parameters of Improved Particle Swarm Optimization}

\section{A. Improved particle swarm optimization algorithm}

The basic PSO algorithm in particle in the whole iterative process is affected by a fixed speed, optimizing the general quality. To this end, Y.Shi suggested that the introduction of the inertia weight of $\omega$ in the velocity update formula to adjust the particle velocity, change into the iterative formula:

$v_{i d}^{k+1}=w v_{i d}^{k}+c_{1} r_{1}\left(p_{i d}-x_{i d}^{k}\right)+c_{2} r_{2}\left(p_{g d}-x_{i d}^{k}\right)$

Inertia weight $\omega$ introduced greatly improves the PSO algorithm optimization performance. The initial inertia weight $\mathrm{W}$ bit constant, later found that the optimization effect is not ideal. Many scholars after the study found that larger inertia weight can improve global search ability of particle, the larger location search, smaller inertia weight $\mathrm{W}$ is conducive to local search of particles, and is beneficial to search for the optimal solution. In the early days of the inertia weight $\omega$ set larger, as the iteration proceeds, $\omega$ gradually is pee on local search, which is commonly used linear decreasing inertia weight, as shown below:

$$
w=w_{\max }-\frac{w_{\max }-w_{\min }}{T_{\max }} T
$$

The $\omega_{\min }$ and $\omega_{\max }$ are $\mathrm{w}$ the minimum and maximum values; $\mathrm{T}$ population the number of iterations, $\mathrm{T}_{\max }$ population the maximum number of iterations.

In order to guarantee the convergence of the algorithm, CLERC in the 90s of the 20th century proposed introducing the constriction factor particle swarm algorithm, by in the velocity updating formula introduced shrinkage factor was proposed to improve the optimization performance. The convergence factor is defined as follows:

$$
\begin{gathered}
v_{i d}^{k+1}=x\left[v_{i d}^{k}+c_{1} r_{1}\left(p_{i d}-x_{i d}^{k}\right)+c_{2} r_{2}\left(p_{g d}-x_{i d}^{k}\right)\right] \\
x=\frac{2}{\left|2-\phi-\sqrt{\phi^{2}-4 \phi}\right|}, \phi=c_{1}+c_{2}, \phi>4
\end{gathered}
$$

Usually set up $c_{1}=c_{2}=2.05, \phi=c_{1}+c_{2}=4.1$, through calculation that $x=0.729$. 
The parameters in PSO algorithm mainly includes: the population size $\mathrm{N}$, the maximum speed threshold $\mathrm{V}_{\max }$, threshold $\mathrm{X}_{\max }$ maximum position, the inertia weight $\mathrm{w}$, cognitive coefficient $C_{1}$ and social factor $C_{2}$. Methods the first three parameters are used to determine the range of numerical experiments. The latter three parameters directly affect the trajectory of particles, and therefore has a more direct impact on the performance of algorithm, this paper mainly studies the three parameters.

\section{B. The method of selecting parameters}

In order to understand the effects of various parameters on particle trajectory in the search process, and to verify the convergence condition of a section, parameter selection of several classical values for particle optimization trajectory simulation, the simulation conditions were: $\mathrm{X}(0)=10, \mathrm{~V}(0)=8, \mathrm{P}=0$, the iteration number is 50 . The results are as shown in Figure 2. In Figure 2 (a) $\mathrm{w}=0.1, \mathrm{P}=0.3$ results, the corresponding eigenvalue is real, non oscillatory convergence; Figure 2 (b) $\mathrm{w}=0.7, \mathrm{P}=0.5$ results, the corresponding eigenvalues with positive real part of the complex.
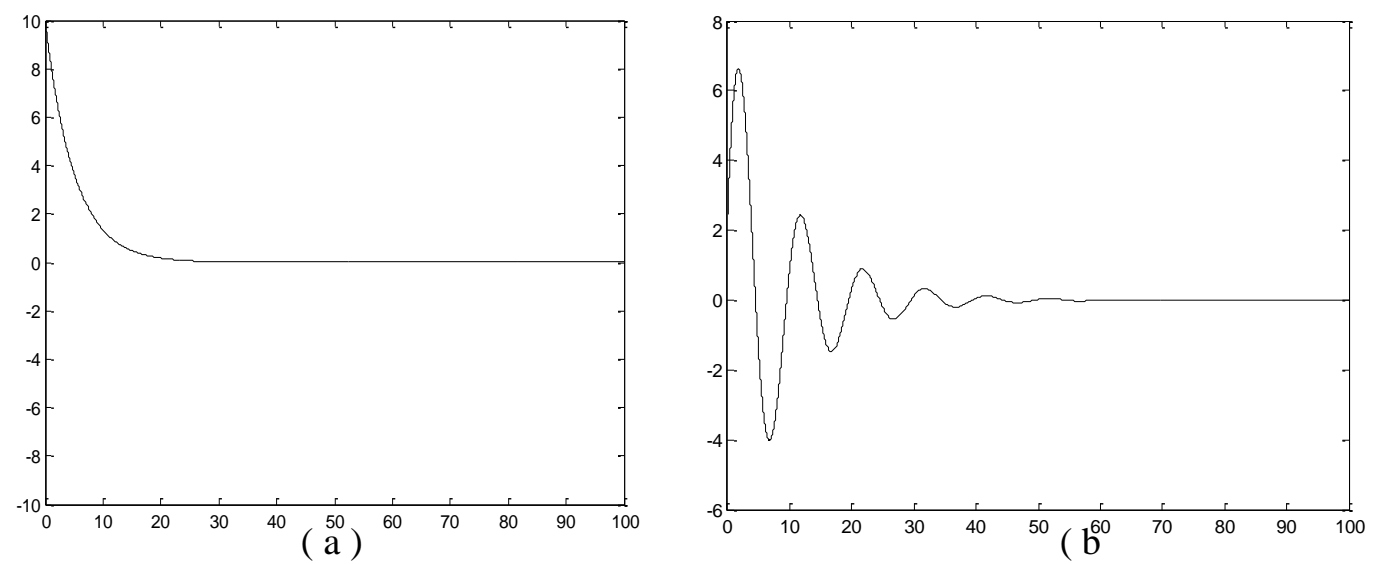

Figure 2. The Results of Several Classical Values for Particle Optimization

According to the formula (11-13) shows that PSO algorithm, particle trajectory is based on Max $(\lambda 1, \lambda 2)$ of the exponential form of convergence or divergence, and the parameter form decides the convergence model of particle trajectories. The different parameters have different optimization results; in fact, even for the same parameter, the random algorithm, may also get different results.

\section{Calculation steps and flow chart}

Reactive power optimization steps of improvement of power system based on particle swarm optimization algorithm:

Step 1: input distribution network data and the parameters of the algorithm, including the upper and lower limits of generator parameter information, distribution line message and control variables the state variables values, such as the control variables of the generator bus voltages UG limit, transformer t step and upper and lower limits and capacitor compensation device capacity QC step, upper limit and lower limit. The constraints of state variables of reactive power output of generator and load node voltage Upg.

Step 2: design iterations $\mathrm{t}=0$, select the appropriate population size $\mathrm{n}$, random initialization of the population in the position of the particle $\mathrm{x}$, and its limit in the range constraint; random initialization of the particle velocity $\mathrm{V}$ and its limit in the range constraint. Set velocity ranges were variable constraints on the lower limit of 1/2.

Step 3: according to the distribution of power flow calculation, the active power and reactive power, voltage and power loss etc.. Calculated active power loss with respect to 
the nodal sensitivity of the reactive power, according to the size of the sensitivity, selected nodes reactive power compensation to reduce the system power loss is most effective compensation point;

Step4: according to the results of power flow calculation to calculate the fitness function value of $\mathrm{F}(\mathrm{x})$. Follow the mixed particle swarm (HPSO) algorithm to find the optimal steps.

Figure 3 is the reactive power optimization flow chart based on HPSO algorithm:

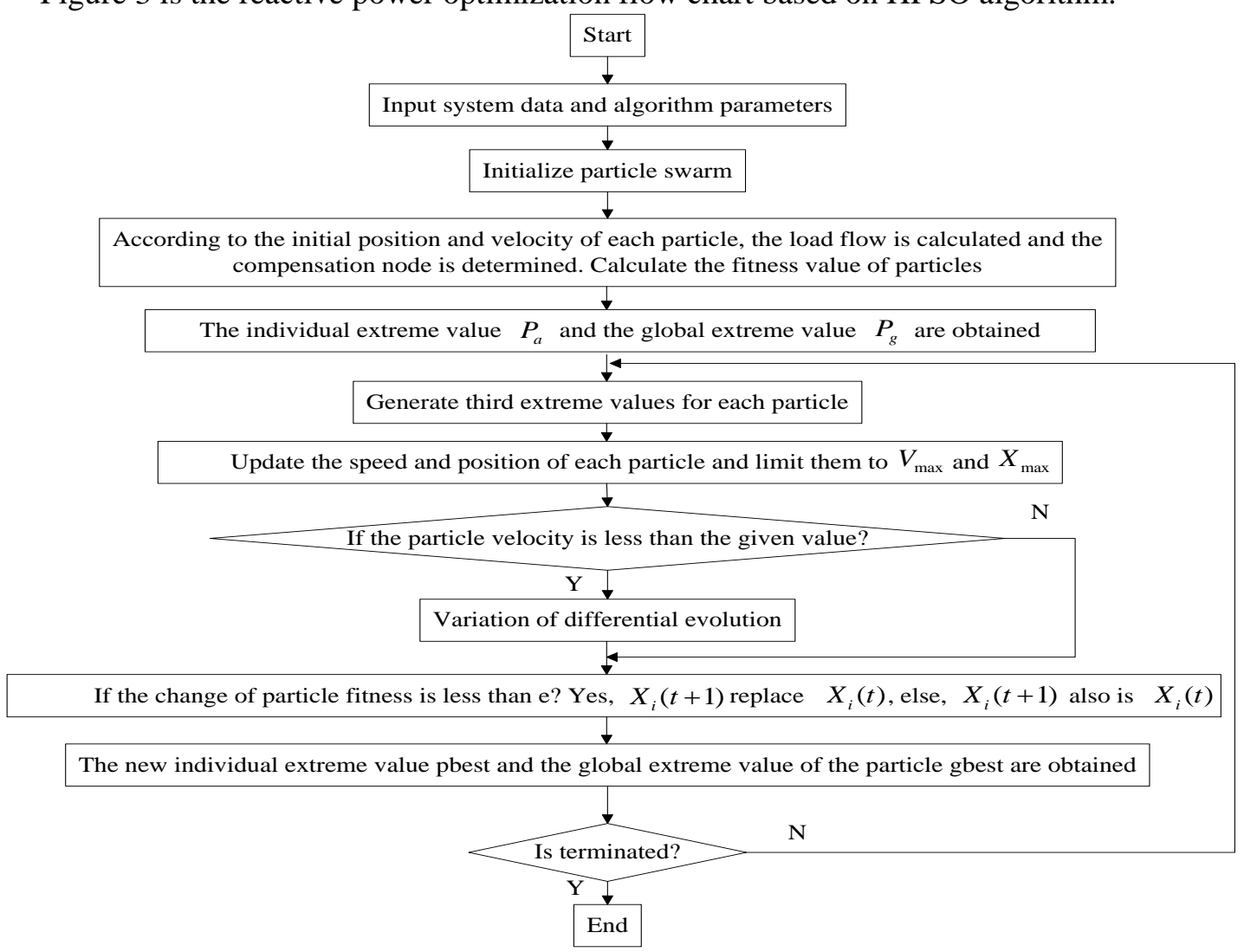

Figure 3. The Reactive Power Optimization Flowing Chart based on the
HPSO Algorithm

\section{Experimental Results}

A. Test result analysis of algorithm

In order to hybrid particle swarm optimization algorithm (HPSO) optimization result analysis, we use the following test functions of the algorithm are tested, the two test functions are:

f1: Rosenbrock function:

$f(x)=\sum_{i=1}^{n}\left(100\left(x_{i+1}-x_{i}^{2}\right)^{2}+\left(x_{i}-1\right)^{2}\right),\left|x_{i}\right| \leq 50$

f2: Rastrigin function:

$f(x)=\sum_{i=1}^{n}\left(x_{i}^{2}-10 \cos \left(2 \pi x_{i}\right)+10\right),\left|x_{i}\right| \leq 5.12$ 
Algorithm parameters are set as follows: the population size $\mathrm{N}=50$, the inertia weight $\omega \max =0.8, \omega \min =0.4$. Learning factor $\mathrm{r} 1$ and $\mathrm{r} 2$ is in the interval $[0,1]$ oral from uniformly distributed random numbers, the maximum number of iterations max. HPSO algorithm and basic PSO algorithm in optimization test functions to value and the number of iterations as shown in Table 1 shows, Figure 4.1 and 4.2 test function $\mathrm{f} 1$ and $\mathrm{f} 2$ adaptation degree and the number of iterations plot.

Table 1. The Contrast of Test of Two Algorithms

\begin{tabular}{clcllll}
\hline & \multicolumn{3}{c}{ PSO } & & & HPSO \\
\hline function & $\begin{array}{l}\text { Average } \\
\text { optimal } \\
\text { value }\end{array}$ & $\begin{array}{l}\text { Optimal } \\
\text { fitness } \\
\text { value }\end{array}$ & $\begin{array}{l}\text { Iteration } \\
\text { number }\end{array}$ & $\begin{array}{l}\text { Average } \\
\text { optimal } \\
\text { value }\end{array}$ & $\begin{array}{l}\text { Optimal } \\
\text { fitness } \\
\text { value }\end{array}$ & $\begin{array}{l}\text { Iteration } \\
\text { number }\end{array}$ \\
$f_{1}$ & 1.3 & 1.0 & 88 & $3.3 \mathrm{e}-03$ & $2.5 \mathrm{e}-03$ & 96 \\
$f_{2}$ & $2.2 \mathrm{e}-01$ & $1.6 \mathrm{e}-01$ & 47 & $5.7 \mathrm{e}-04$ & $4.2 \mathrm{e}-04$ & 97 \\
\hline
\end{tabular}
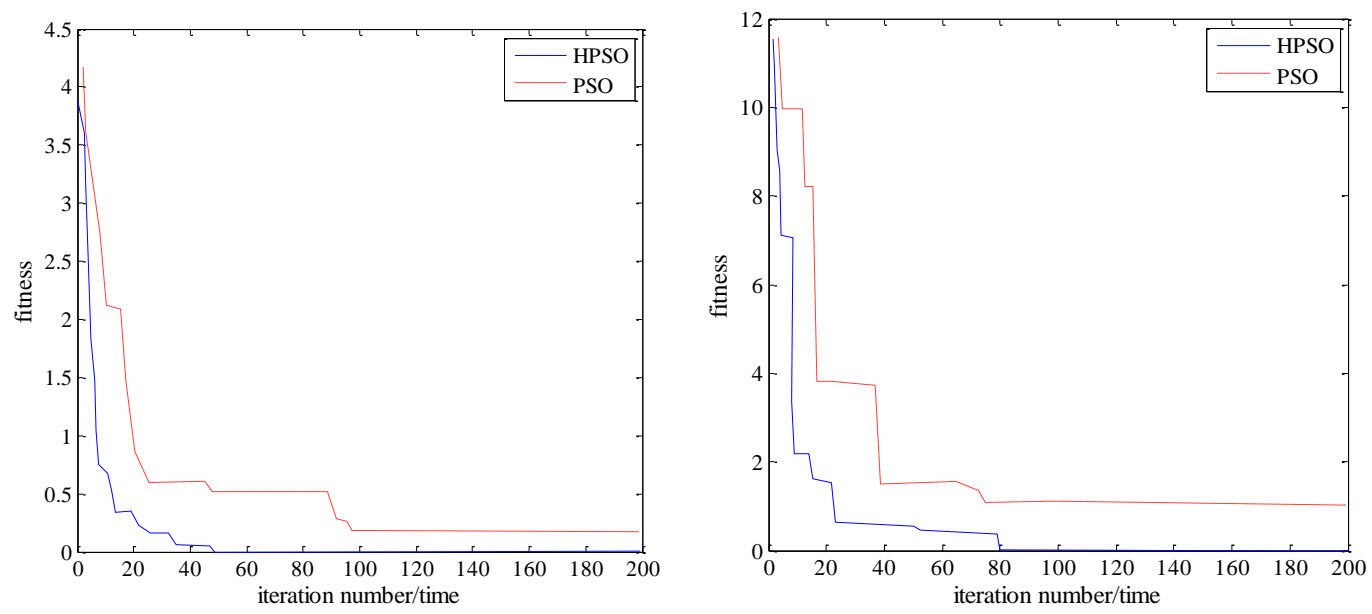

Figure 4. Test Function f1 and f2 Adaptation Degree and the Number of Iterations Plot

Can see hybrid particle group optimization algorithm regardless of from the calculation precision and calculation speed are higher than the basic particle swarm algorithm, keep the basic particle swarm optimization algorithm, the concept of simple, easy to achieve some of the advantages, while avoiding the algorithm is easy to fall into local optimum, is a more practical optimization algorithm.

\section{B. Experimental results and analysis}

In order to test hybrid particle swarm algorithm is used to solve the problem of reactive power optimization is effective and the matlab7.0 platform written based on GA and PSO, HPSO algorithm without reactive power optimization program, and the simulation calculation of IEEE14 and IEEE30 bus system is done. Finally, the optimization results were analyzed and compared. The system parameters are the standard value, the voltage phase angle is in radians, reference power is set to 100MVA.

Figure 5.1 for the reactive power optimization and node voltage contrast curves based on three kinds of algorithms. Figure 5.2 shows the three algorithms in reactive power optimization curve fitness function calculation process. 

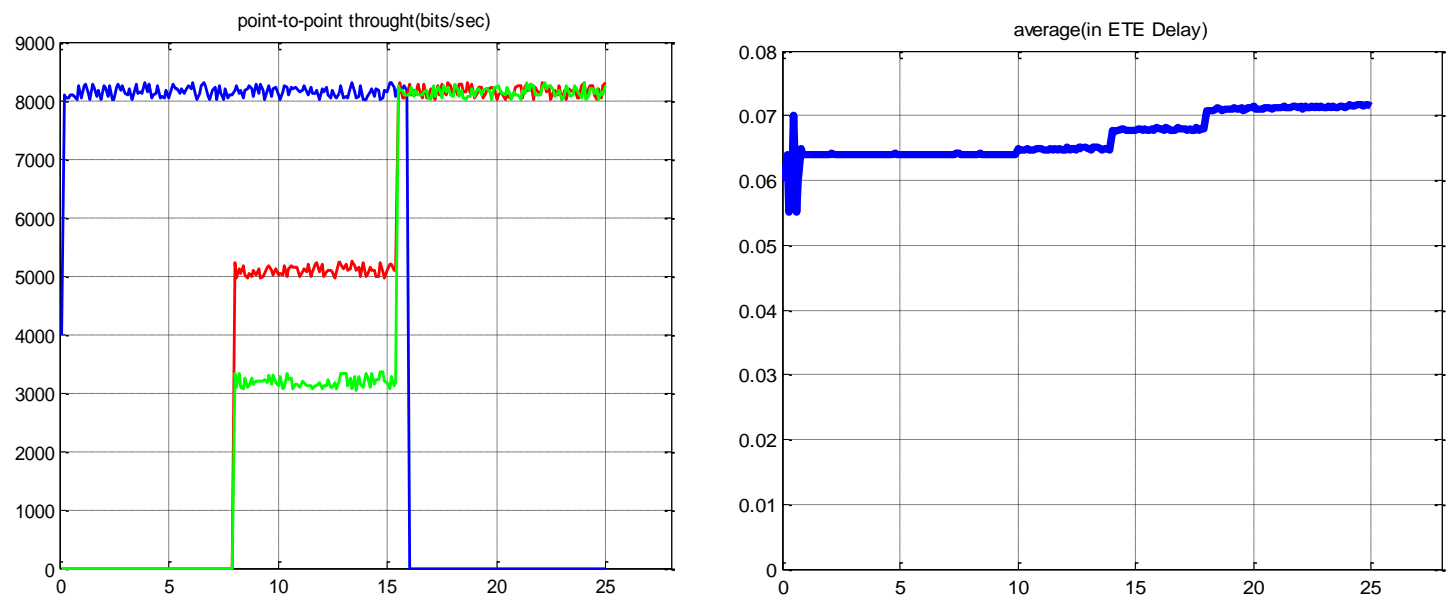
Figure 5. The Reactive Power Optimization and
Node Voltage Contrast Curves

Hybrid particle swarm optimization algorithm (HPSO) is applied to the power system reactive power optimization. It gives the HPSO algorithm in solving the problem of reactive power optimization detailed steps and flow chart. Improved particle swarm optimization algorithm is used to validate the effectiveness through the nodes of the system simulation calculation, results show that: algorithm is proposed in this paper is feasible, through comparing with the genetic algorithm and basic particle swarm optimization (PSO) derived, based on HPSO algorithm of reactive power optimization in calculation speed and search accuracy is higher than that of the above two algorithms, which not only reduces the power loss of the system, and improve the voltage quality of the whole system, has good practicability.

\section{Conclusions}

The domestic and foreign power system without reactive power optimization research present situation to carry on the research and analysis the no reactive power control method, and established the system of minimum network loss as the objective function of the mathematical model. The basic particle swarm algorithm were research and analysis, which combines the differential evolution algorithm and simulated annealing algorithm and particle swarm optimization algorithm, a new hybrid particle swarm optimization algorithm (HPSO) is proposed. The HPSO algorithm combines the advantages of three algorithms, has good search performance. For control variables both continuous variables and discrete variables characteristics, adopt hybrid coding method to encode the variables. At the same time, the mapping and four to five warping method dealing with the coexistence of continuous and discrete variables homes.

\section{Acknowledgements}

The authors wish to acknowledge the Key Disciplines Foundation of electronic science and technology of Qianjiang College and the Internet of Things (IOT) engineering of Hangzhou city for their support. 


\section{References}

[1] V.C. Gungor, F.C. Lambert, "A survey on communication networks for electric system automation, Computer Networks”, vol. 50, no. 7, (2006),pp. 877-897

[2] N. Papandreou, T. Antonakopoulos, "Resource allocation management for indoor Power-line communications systems", IEEE Transactions on Power Delivery, (2007), vol. 22, no. 2, pp. 893-90.

[3] A. Angel, B. Rujula, "Future development of the electricity systems with distributed generation", Energy, (2009), vol. 34, no. 3, pp.377-383

[4] Q. Bing, Y. Xuc-jinl, G. Gang-junl, "Design and realization of electric Power communication resource management system", Relay, vol. 33, no. 16, (2005), pp. 54-61.

[5] E. Luder, "The network-and system's theory of electrical communications", Umschau inWissensehaft und Teehnik, vol. 74, no. 10, (1974), pp. 303-306

[6] J. Cai, X. Shen, J. W Mark, "Downlink resource management for packet transmission in OFDM wireless communication systems", IEEE Transactions on Wireless Communications, vol. 4, no. 4, (2005), pp. 688-1702.

[7] L. Zhao, J.W. Mark, "Integrated Power control and rate allocation for radio resource management in uplink wideband CDMA systems", Proceedings. Sixth IEEE International Symposium on a World of Wireless Mobile and Multimedia Networks, (2005),pp. 428-436

[8] M. C. Kim, J. Park, W. Jung, H. Kim, Y. J. Kim, "Development of a standard communication protocol for an emergency situation management in nuclear power plants", Annals of Nuclear Energy, (2010), vol. 37, no. 6, pp. 888-893

[9] C.-H. Lien, H.-C. Chen, Y.-W. Bai, M.-B. Lin, "Power Monitoring and Control for Electric Home Appliances Based on Power Line Communication", Instrumentation and Measurement Technology Conference proccedings, (2008), pp. 2-79.

[10] L. Xu, T. Chen, Z. Ren, D. Wu, "Resource management for uplink OFDM wireless communication system", Global Mobile Congress, (2007), pp. 284-288

[11] M. Khodier, G. Saleh, "Beamforming and Power control for interference reduction in wireless communications using particle swarm optimization", International Journal of Electronics and Communications, vol. 64, no. 6, (2010), pp. 489-502

[12] A. Radonjie, V. Vujicie, "Integer SEC-DED codes for low power communications", Information Processing Letters, (2010), vol. 110, pp. 518-520.

[13] I. Auge, F. Petrot, F. Donnet and P. Gomez, "Platform-based design from parallel C specifications", IEEE Transactions on Computer-Aided Design of Integrated Circuits and Systems, vol. 24, no. 12, (2012), pp. 1811 - 1826.

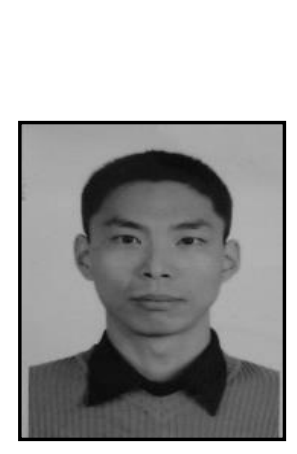

\section{Authors}

Wang Yu-huai, He works currently as a Lecturer of Electronic Information Engineering in Qianjiang College of Hangzhou Normal University. He has achieved the M.S. degree in mechatronic engineering from Zhejiang University of Technology in 2006. He is a $\mathrm{PhD}$ candidate in plastic forming. His major interest work area is Computer Application Technology and Machine Vision, Incremental Forming.

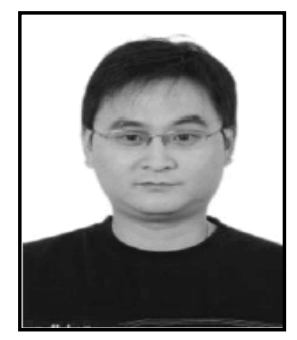

Wang Qi-hui, He works currently as a computer teacher in Qianjiang College of Hangzhou Normal University. He has achieved the M.S. degree of Computer Science and Technology from Zhejiang University in 2002. He is a PH.D Candidate in the School of Aeronautics and Astronautics at Zhejiang University. His major interest work area is Computer Vision, Computer Graphics and Image Processing. 

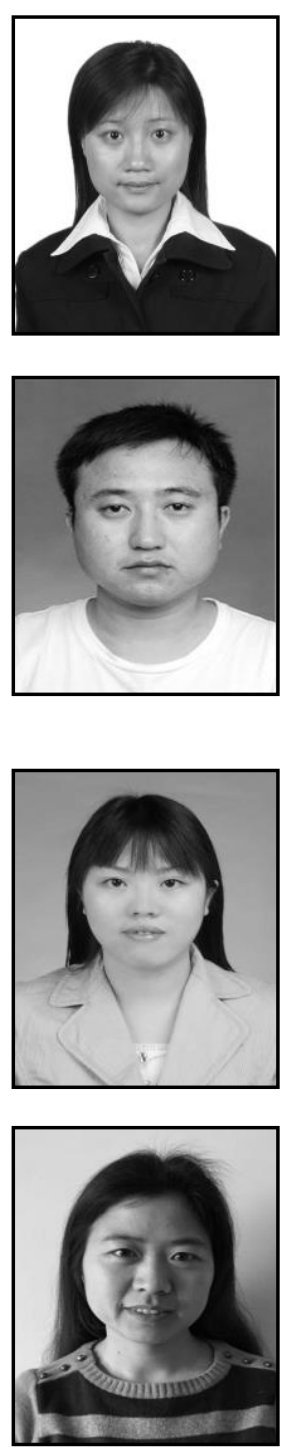

Zhang Hui-xi, She works currently as a Lecturer of Electronic Information Engineering in Qianjiang College of Hangzhou Normal University. She has achieved a master's degree of Electronic Circuit and System from Zhejiang University. His major interest work area is Digital Systems Development, Data Acquisition and Signal Processing.

An Kang, $\mathrm{He}$ works currently as a Lecturer of Electronic Information Engineering in Qianjiang College of Hangzhou Normal University. She has achieved a master's degree of Electronic Circuit and System from Guilin University of electronic technology. His major interest work area is Wireless Sensor Networks , MIMO Wireless Communication and Heterogeneous Networks Vertical Handoff .

Ye Xia, She works currently as an electronic information engineering teacher in Qianjiang College of Hangzhou Normal University. She has achieved a master's degree of Communication and information system from Chongqing University of Post and Telecommunications. Her main research interests include embedded processor architecture and real-time operating system.

Sun Ya-ping, She works currently as an electronic information engineering teacher in Qianjiang College of Hangzhou Normal University. She has achieved a master's degree of Power Electronics from Nanjing University of Aeronautics and Astronautics. Her major interest work area is Power Electronics, Power Drive and Digital Power. 
International Journal of Grid and Distributed Computing Vol. 9, No. 1 (2016) 